\title{
Comprehensive analysis of a long non-coding RNA-associated competing endogenous RNA network in glioma
}

\author{
JIE ZHU ${ }^{1 *}$, HAN WANG $^{2 *}$, YUE-QING HUANG ${ }^{3 *}$, WEI SONG ${ }^{4}$, \\ YI-FAN LI ${ }^{5}$, WEN-JIE WANG ${ }^{6}$ and ZHI-LIANG DING ${ }^{7}$

\begin{abstract}
${ }^{1}$ Department of Oncology, Changzhou Traditional Chinese Medical Hospital, Changzhou, Jiangsu 213003;
${ }^{2}$ Department of Oncology, Jining Cancer Hospital, Jining, Shandong 272000; Departments of ${ }^{3}$ General Practice and ${ }^{4}$ Intervention and Vascular Surgery, The Affiliated Suzhou Hospital of Nanjing Medical University, Suzhou, Jiangsu 215001;

${ }^{5}$ Department of Oncology, Binzhou People's Hospital, Binzhou, Shandong 256600; Departments of ${ }^{6}$ Radio-Oncology and

${ }^{7}$ Neurosurgery, The Affiliated Suzhou Hospital of Nanjing Medical University, Suzhou, Jiangsu 215001, P.R. China
\end{abstract}

Received October 3, 2019; Accepted April 9, 2020

DOI: $10.3892 / \mathrm{ol} .2020 .11924$

\begin{abstract}
Long non-coding RNAs (lncRNAs) can act as competing endogenous RNAs (ceRNAs), interacting with microRNAs (miRNAs) and playing an important role in tumor progression. However, the role of IncRNA-mediated ceRNAs in glioma remains largely unknown. The present study aimed to identify novel lncRNAs and their associated function in glioma. RNA sequencing and corresponding clinical data from patients with glioma were obtained from The Cancer Genome Atlas. A total of 598 glioma tissues and 5 normal brain tissues were analyzed in the present study. The differentially expressed (DE) IncRNAs, mRNAs and miRNAs were identified using R packages and were used to construct a ceRNA network. Gene Ontology and Kyoto Encyclopedia of Genes and Genomes analyses were performed to investigate the biological functions of the DEmRNAs. Kaplan-Meier curve analysis was performed to investigate the association between DElncRNA expression and patient outcome. A total of 752 DElncRNAs, 2,079 DEmRNAs and 113 DEmiRNAs were identified between glioma and normal tissues. A lncRNA-miRNA-mRNA ceRNA network consisting of 61 lncRNAs, 12 miRNAs and 92 mRNAs was constructed. Survival analysis indicated that 36 DElncRNAs, 72 DEmRNAs and 3 DEmiRNAs were associated with overall
\end{abstract}

Correspondence to: Dr Zhi-Liang Ding, Department of Neurosurgery, The Affiliated Suzhou Hospital of Nanjing Medical University, 16 Baita West Road, Suzhou, Jiangsu 215001, P.R. China E-mail: dr_zhiliangdin@163.com

Dr Wen-Jie Wang, Department of Radio-Oncology, The Affiliated Suzhou Hospital of Nanjing Medical University, 16 Baita West Road, Suzhou, Jiangsu 215001, P.R. China

E-mail: doctor.wjwang@gmail.com

*Contributed equally

Key words: glioma, competitive endogenous RNA, long non-coding RNA, survival analysis survival in patients with glioma. The present study identified novel lncRNAs associated with survival prognosis and may facilitate further investigation of IncRNA-mediated ceRNA regulatory mechanisms in glioma.

\section{Introduction}

Primary brain and central nervous system (CNS) tumors have a mortality of $7 \%$ in patients $<70$ years old, and an estimated 270,000 non-malignant and 120,000 malignant tumors were reported between 2011 and 2015 in the USA (1,2). In 2017, $\sim 80,000$ new cases and 17,000 primary brain tumor-related mortalities were reported (3). Furthermore, one-third of the cases were diagnosed as malignant tumors (4). Glioma is a broad term that encompasses several types of malignant tumor derived from neuroepithelial cells, such as glial cells and other supporting cells of the CNS $(1,5,6)$. Gliomas account for $\sim 80 \%$ of primary brain tumors in adults and can be categorized as astrocytomas, oligodendrogliomas, ependymomas, mixed gliomas and other rare types, including brain stem and optic nerve gliomas (7). According to the World Health Organization criteria, gliomas vary histopathologically and may include benign ependymomas, as well as the most aggressive grade-IV glioblastoma (GBM) (5). Surgical removal is the primary therapy for intracranial tumors. However, tumors located in inoperable or sensitive regions of the brain pose clinical challenges (8). In addition, limited effectiveness of systemic chemotherapy can be attributed to the blood-brain barrier, which protects the brain from harmful compounds but also restricts the entry of chemotherapeutic drugs (9). The molecular mechanisms underlying the development of gliomas remain largely unknown. Therefore, elucidating the associated mechanisms may accelerate the development of targeted therapies for the disease.

The competing endogenous RNA (ceRNA) hypothesis, proposed by Salmena et al (10), has provided insight for RNA regulatory networks. According to this hypothesis, mRNA, long non-coding RNA (lncRNA), pseudogenes and other molecules can competitively bind to the same microRNA (miRNA/miR) response element and modulate miRNA function, forming an RNA regulatory network (10). 

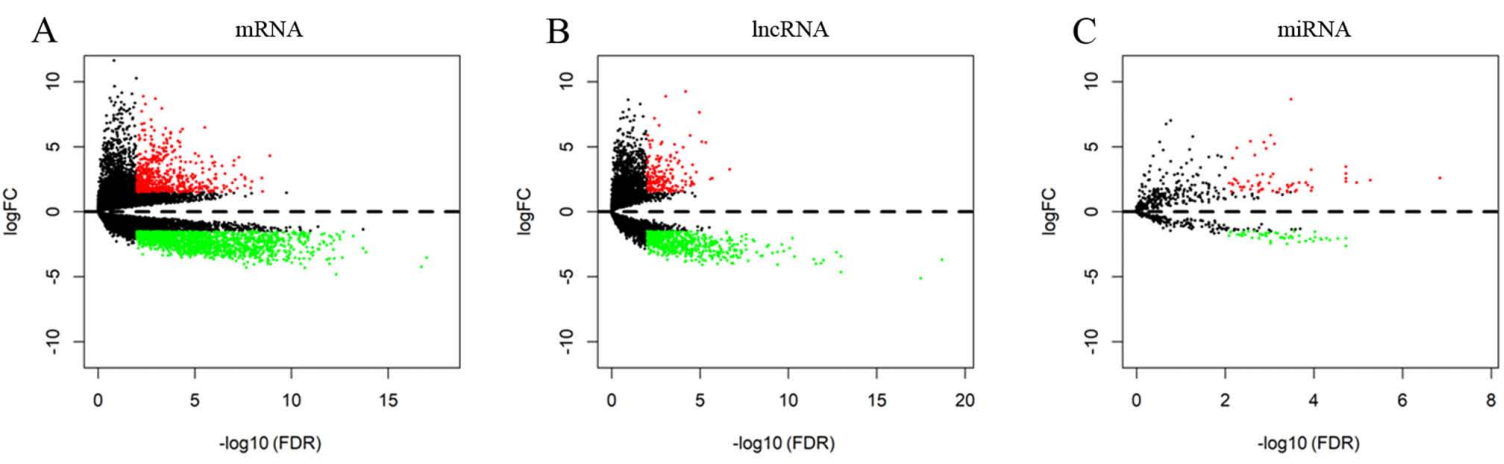

Figure 1. Volcano plot of differentially expressed (A) mRNAs, (B) lncRNAs and (C) miRNAs. Red dots represent upregulated RNAs and green dots represent downregulated RNAs with statistical significance. $\mathrm{P}<0.01$ indicated a statistically significant difference. lncRNAs, long non-coding RNAs; miRNAs, microRNAs; FDR, false discovery rate; FC, fold change.

IncRNAs are RNAs $>200$ bp in length that do not encode proteins (11). Previous studies suggested that lncRNAs are a by-product of RNA transcription with biological functions that include modulation of the nervous system at the epigenetic, transcriptional and post-transcriptional levels (12-14). Additionally, lncRNAs serve as ceRNAs and participate in regulating gene expression and encoding miRNAs $(10,15)$. Furthermore, lncRNAs were demonstrated to serve an important role in oncogenesis and tumor progression (16).

The present study constructed a ceRNA network in order to investigate and to identify potential biomarkers for glioma, and to determine the molecular mechanisms of glioma pathogenesis, using Gene Ontology (GO) and Kyoto Encyclopedia of Genes and Genomes (KEGG). Additionally, the prognostic value of the identified mRNAs, miRNAs and lncRNAs was analyzed by survival analysis.

\section{Materials and methods}

Data acquisition and processing. RNA sequencing and the corresponding clinical data of patients with glioma (low-grade glioma and glioblastoma) were obtained from The Cancer Genome Atlas (TCGA) data portal (https://tcga-data.nci.nih. gov/tcga/) (17). The lncRNA, mRNA and miRNA sequence data were derived from the Illumina HiSeq platform (Illumina, Inc.). A total of 598 glioma tissues and 5 normal brain tissues were included in the present study. DESeq package in $\mathrm{R}$ software was confirmed to identify significant DEGs in the study (https://bioconductor.org/packages/release/bioc/html/DESeq. html)(18). The present study was conducted in accordance with the publication guidelines provided by TCGA (http://cancergenome. nih.gov/publications/publicationguidelines). Therefore, further approval from the local ethics committee was not required.

Identification of differentially expressed (DE) RNA. DElncRNAs and DEmRNAs were defined and encoded based on the annotations from the Ensembl database (http://www.ensembl.org/index.html). Using the edgeR package version.3.53 in $\mathrm{R}$ language to further analyze the data, the DElncRNAs, DEmRNAs and DEmiRNAs were identified (https://www.r-project.org). $\mid \log _{2}$ fold change (FC) $>1.5$ and false discovery rate (FDR) adjusted to $\mathrm{P}<0.01$ were set as the thresholds. In addition, heat maps and volcano maps of the
DE RNAs were generated, using the gplots and heatmap R packages v3.53 (https://www.r-project.org).

ConstructionoftheceRNAand cytohubbanetworks. ThemiRcode (http://www.mircode.org/) database and the Perl program (http://www.perl.org) were used to predict lncRNA-miRNA interactions, and the miRNAs sequences were identified by using StarBase version.2.0 database (http://starbase.sysu.edu. $\mathrm{cn} /$ ). miRNA-targeted mRNAs were retrieved from the miRDB (http://mirdb.org/), miRTarBase version.7.0 (http://mirtarbase. mbc.nctu.edu.tw/php/index.php) and TargetScan databases version.7.2 (http://www.targetscan.org/vert_72/) (19-21). miRTarBase, miRDB, and TargetScan were used to identify the target genes of miRNAs. Only mRNAs recognized by all three databases were considered as candidate mRNAs and intersected with the DEmRNAs to screen out the DEmRNAs targeted by the DEmiRNAs. A co-expression network of DE genes (DEGs) was then constructed, based on DEmiRNA-DElncRNA and DEmiRNA-DEmRNA interactions, which were visualized using Cytoscape version.3.61 (National Institutes of Health). The cytohubba plugin (22) was used to evaluate the top-10 network in the network based on the degree of association between RNAs, which was termed as closeness.

Functional enrichment analysis. To examine the underlying biological mechanisms of DEmRNAs in the ceRNA crosstalk network, GO annotation and KEGG pathway analyses were conducted using the DAVID version.6.7) (https://david. ncifcrf.gov/) online tool (23) and cluster Profiler (https://www. rdocumentation.org/packages/clusterProfiler/versions/3.0.4), an $\mathrm{R}$ package for functional classification and enrichment of gene clusters using hypergeometric distribution. The GO plot package of R software was utilized to display the results of the GO and KEGG analyses (http://wencke.github.io/). GO and KEGG enrichment analysis was based on the threshold of $\mathrm{P}<0.05$.

Survival analysis. To assess the prognostic value of DElncRNAs in patients with glioma, survival analysis for these DERNAs in the ceRNA network was conducted using the survival package in $\mathrm{R}$ (https://www.rdocumentation. org/packages/survival/versions/2.42-3). Survival curves were generated using the Kaplan-Meier method and the log-rank test was used to compare the difference between the groups. 

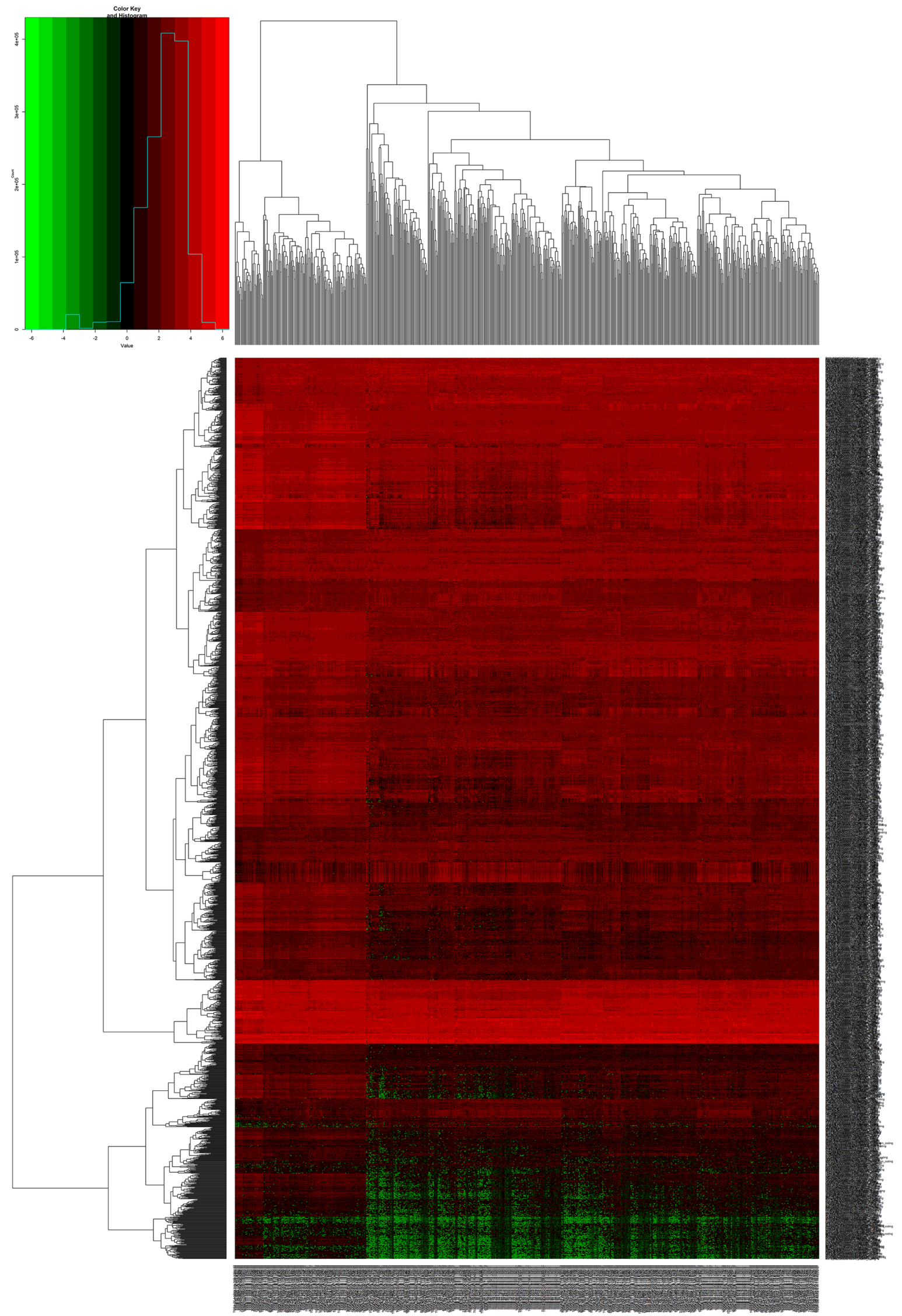

Figure 2. Heatmap of differentially expressed mRNAs between glioma and non-tumor tissues.

Univariate Cox regression analyses were performed to independently identify the effects of DEmRNAs, DElncRNAs and
DEmiRNAs on overall survival (OS). $\mathrm{P}<0.05$ was considered to indicate a statistically significant difference. 


\section{Results}

DElncRNAs, DEmiRNAs and DEmRNAs in glioma. Using the 'DESeq' package in R software, significant DEGs in 598 glioma tissues and 5 normal brain tissues were identified. A total of 752 DElncRNA (180 upregulated and 572 downregulated), 2,079 DEmRNAs (588 upregulated and 1,491 downregulated) and 113 DEmiRNAs (62 upregulated and 51 downregulated) were identified with thresholds of $\log _{2} \mathrm{FCl}>1.5$ and adjusted $\mathrm{P}<0.01$. The distribution of all DEGs on the two dimensions of $-\log$ FDR and $\log F C$ are depicted in the volcano map in Fig. 1. For the heatmaps presented in Figs. 2-4, the numerical data represent the expression profiles of DEGs.

Construction of the ceRNA network. To further examine how lncRNAs interact with miRNAs to regulate mRNA in glioma, a IncRNA-miRNA-mRNA (ceRNA) network was constructed based on the aforementioned data and visualized using Cytoscape v3.6.1. Using the 752 DElncRNAs retrieved from the miRcode database, the Perl program was applied to identify 211 pairs of interacting lncRNAs and miRNAs. Targeted mRNAs were screened based on the 12 miRNAs using the miRTarBase, miRDB and TargetScan database. The final DEmiRNA targeted genes were selected, which were included in all 3 datasets (miRTarBase, miRDB and TargetScan). miRNA-targeted mRNAs not included in DEmRNAs were discarded. Finally, 92 DEmRNAs were included in the ceRNA network (Fig. 5). Thus, a total of 61 lncRNA nodes, 12 miRNA nodes and 92 mRNA nodes as differentially expressed profiles were presented in the ceRNA network (Fig. 6).

Construction of the cytohubba network. Based on the cytohubba plugin, the top-10 network was obtained (Fig. 7). The network consisted of 6 miRNAs, including hsa-mir-93, hsa-mir-424, hsa-mir-497, hsa-mir-195, hsa-mir-182 and hsa-mir-96, and 4 lncRNAs, AC092171.1, family with sequence similarity 87 member A (FAM87A), deleted in lymphocytic leukemia 1 (DLEU1) and lymphocyte antigen 86 antisense RNA 1 (LY86-AS1).

Functional analysis of DEmRNAs in the ceRNA network. The biological functions of the 92 DEmRNAs were further explored using GO and KEGG analysis, which demonstrated that these DEmRNAs were enriched in 42 GO biological process categories $(\mathrm{P}<0.05)$. In the $\mathrm{GO}$ analysis, a total of 5 significantly enriched pathways were obtained (Table I; Fig. 8). The most enriched GO term was 'protein binding'. In the KEGG pathway analysis, a total of 9 significantly enriched pathways were obtained (Table II; Fig. 9); Among the 9 pathways, 'cell cycle', 'dopaminergic synapse' and 'Kaposi sarcoma-associated herpesvirus infection' were linked with the progression of glioma. Additionally, other pathways such as 'relaxin signaling pathway', 'cellular senescence' and 'human T-cell leukemia virus 1 infection' were also tumor-related pathways.

Survival associated IncRNAs in the ceRNA network. To identify the association between DElncRNAs in the ceRNA network and the prognosis of patients with gliomas, a Kaplan-Meier survival analysis was conducted. A total of 36 out of 752
DElncRNAs were significantly associated with OS. Among the 36 significant DElncRNAs, 13 lncRNAs, including DLEU1, dopamine $\beta$-hydroxylase antisense RNA1 (DBH-AS1), hyaluronan synthase 2 antisense RNA1 (HAS2-AS1), LINC02875, AL117190.1, chromosome 9 open reading frame 147, cytochrome P450 family 1 subfamily B member 1 antisense RNA 1, human leukocyte antigen group (HCG) 15, HCG23, LINC00466, tryptophanyl tRNA synthetase 2, maternally expressed (MEG) 3 and MEG8, were negatively associated with OS. The remaining 23 lncRNAs [CCD26 lncRNA, long intergenic non-protein coding RNA (LINC00320), AC011374.1, AC020907.2, AC022400.1, AC092171.1, AC110491.1, ArfGAP with coiled-coil, ankyrin repeat and $\mathrm{PH}$ domains 2 intronic transcript 1, adenosine deaminase RNA specific B2 antisense RNA 1, AL359541.1, AL772363.1, Rho GTPase activating protein 31 antisense RNA 1, ZNF22 antisense RNA 1, calcium voltage-gated channel subunit $\alpha 1 \mathrm{C}$ intronic transcript, glutamate metabotropic receptor 5 antisense RNA 1, LINC00501, MIR4500HG, versican antisense RNA 1, helicobacter pylori responsive 1, LINC00461, myocardial infarction associated transcript (MIAT), small nucleolar (sno) RNA host gene 1 and MIR600HG)] were positively associated with OS. The top 6 most significant survival-associated DElncRNAs are presented in Fig. 10.

\section{Discussion}

Non-coding RNAs include several types of RNAs, and can be classified into lncRNAs and short non-coding RNAs. The latter can be further categorized into transfer RNAs, ribosomal RNAs, miRNAs, small interfering RNAs and snoRNAs (24). Previous studies demonstrated that lncRNAs played a key role in gene transcriptional control, epigenetic regulation and post-transcriptional modification $(25,26)$. The present study analyzed RNA sequencing and clinical data from patients with gliomas acquired from TCGA in order to investigate the functions of lncRNAs in the ceRNA network. DElncRNAs, DEmRNAs and DEmiRNAs were identified using $R$ packages and were used to construct a ceRNA network. The biological functions of the DEmRNAs were determined by GO and KEGG analyses. Further survival analysis was performed to investigate the association between DElncRNAs and OS.

miRNAs are involved in the regulation of a number of biological processes, including cell differentiation, proliferation and apoptosis, by binding with target mRNAs at the transcriptional or post-transcriptional levels (27). A previous study demonstrated that aberrantly expressed miRNAs were closely associated with several types of cancer (28). Moreover, growing evidence suggests that several miRNAs are implicated in the progression of glioma (29-31). hsa-mir-183 is frequently methylated and was correlated with poor outcome in hepatocellular carcinoma (32). Moreover, hsa-miR-183-5p was involved in cell cycle regulation via the MAPK and glioma signaling pathways (33). In addition, multivariative analysis revealed that low serum hsa-mir-137 levels were closely associated with high clinical grades and poor survival in patients with GBM (34). Moreover, hsa-miR-93-5p upregulation was correlated with poor prognosis in non-small cell lung cancer (35), while hsa-miR-93-3p was recognized as a diagnostic biomarker in triple negative breast cancer (36). 

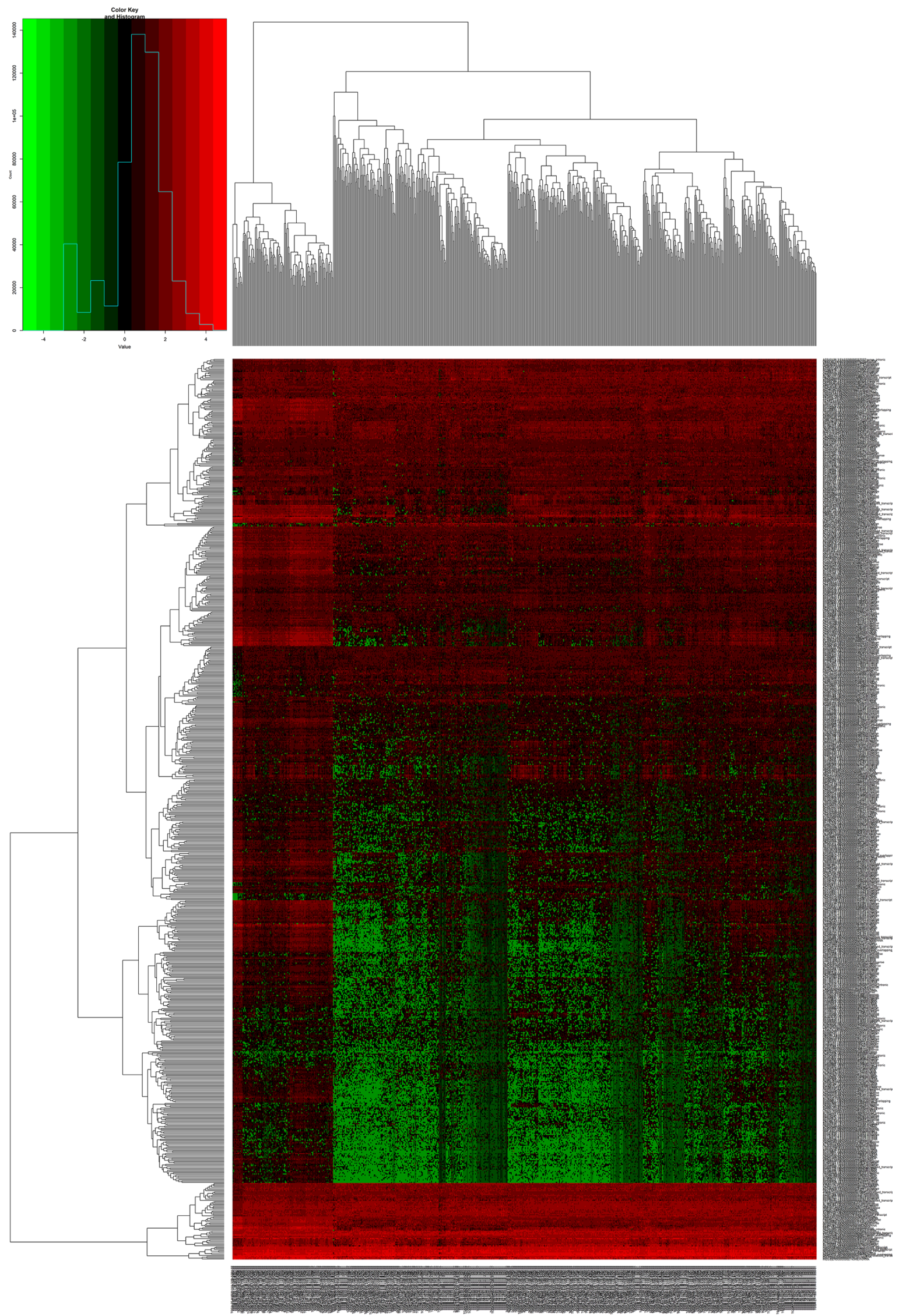

Figure 3. Heatmap of differentially expressed long non-coding RNAs between glioma and non-tumor tissues.

Overexpression of hsa-mir-497 promoted the proliferation of glioma cells (37) and has been demonstrated to be an unfavorable prognostic biomarker in human glioma (38). In the present study, 6 DEmiRNAs, which may serve as 

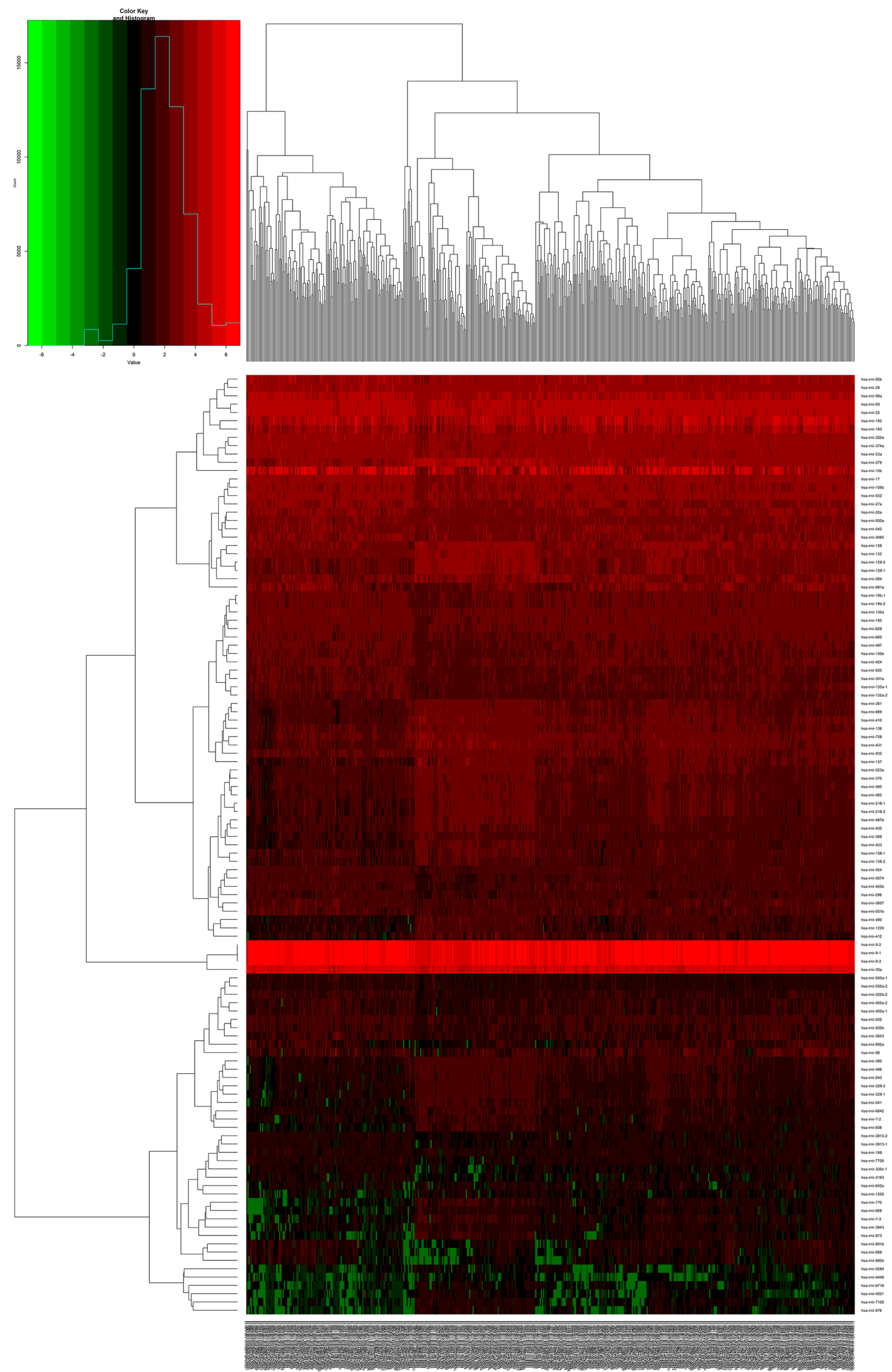

Figure 4. Heatmap of differentially expressed microRNAs between glioma and non-tumor tissues.

promising prognostic biomarkers, were involved in the ceRNA network.
In the present study, 92 DEmRNAs were identified in the ceRNA network. The biological processes and pathways of 


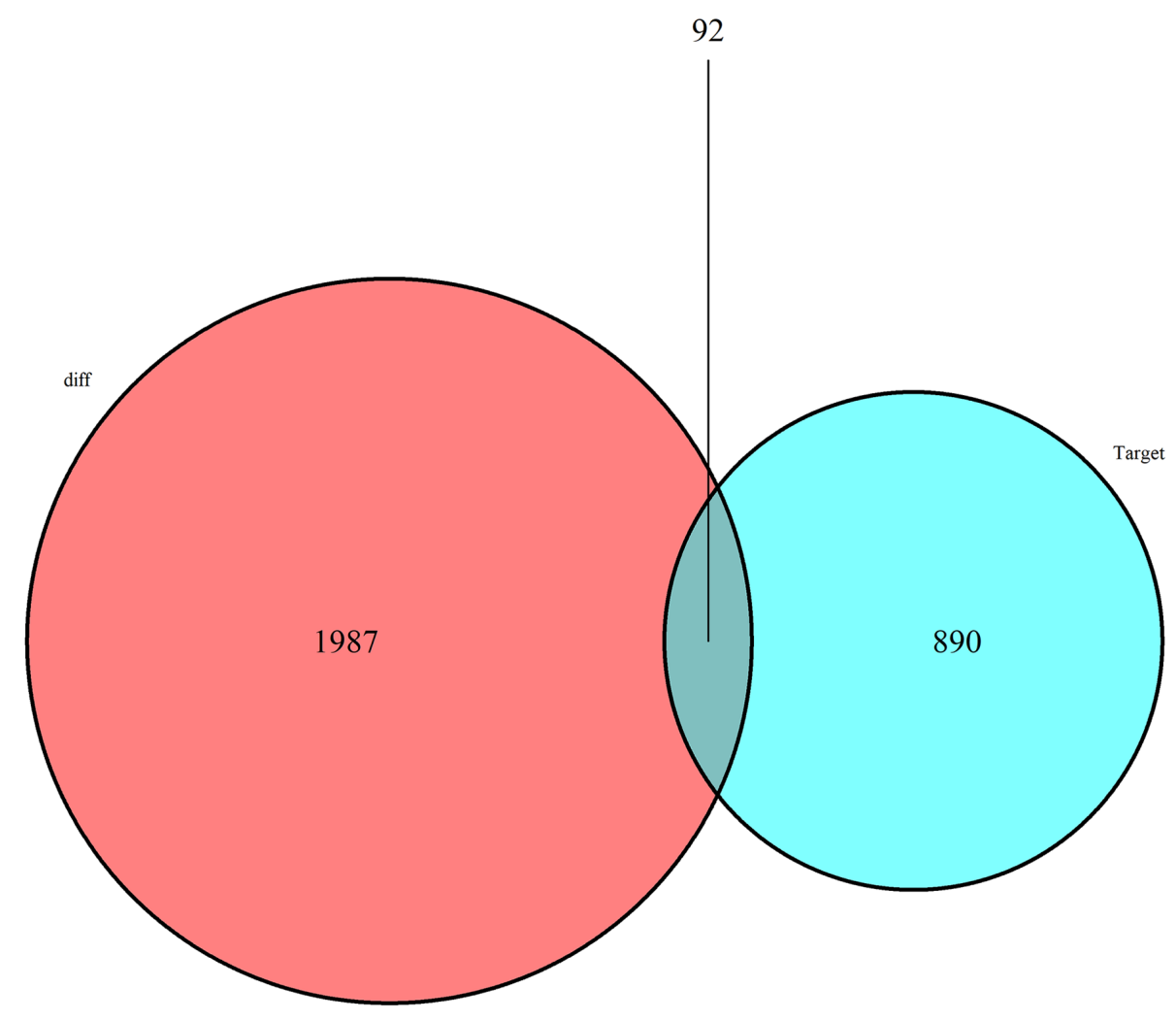

Figure 5. Venn diagram of differentially expressed mRNAs involved in the competing endogenous RNA network. A total of 92 DEmRNAs were included in the ceRNA network. Red, differentially expressed mRNAs; blue, target genes of miRNAs included in miRTarBase, miRDB and TargetScan databases; intersection, DEmRNAs.

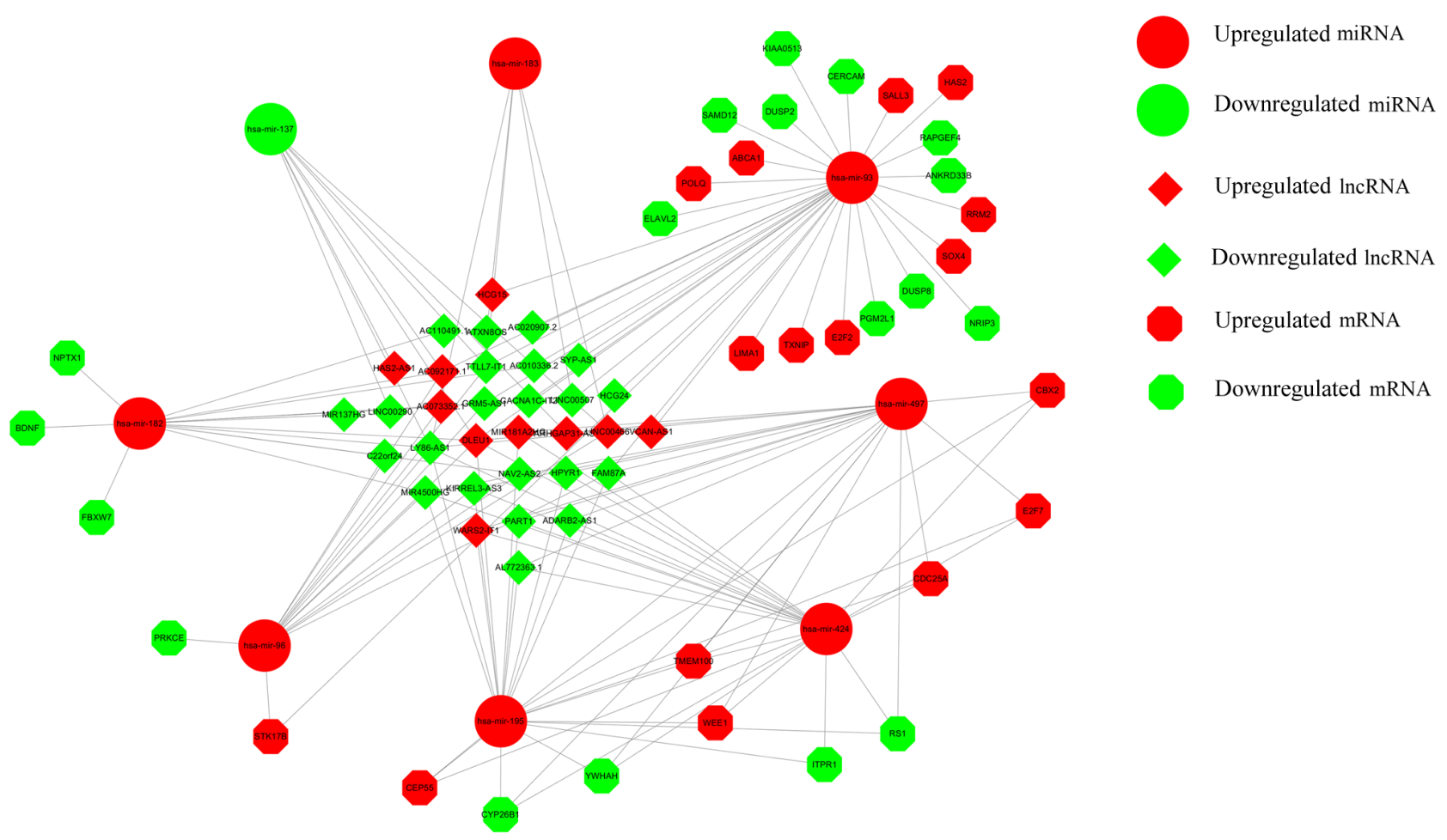

Figure 6. Competing endogenous RNA network of lncRNA-miRNA-mRNA in glioma. lncRNA, long non-coding RNA; miRNA, microRNA.

the DEmRNAs were assessed by GO and KEGG pathway analyses. The most enriched GO biological processes included 'protein binding', 'signal transducer activity', 'protein kinase activity' and 'regulation of cell cycle'. The pathway analysis revealed that several genes were involved in cancer-related pathways, such as 'cell cycle', 'cellular senescence', 'Kaposi 


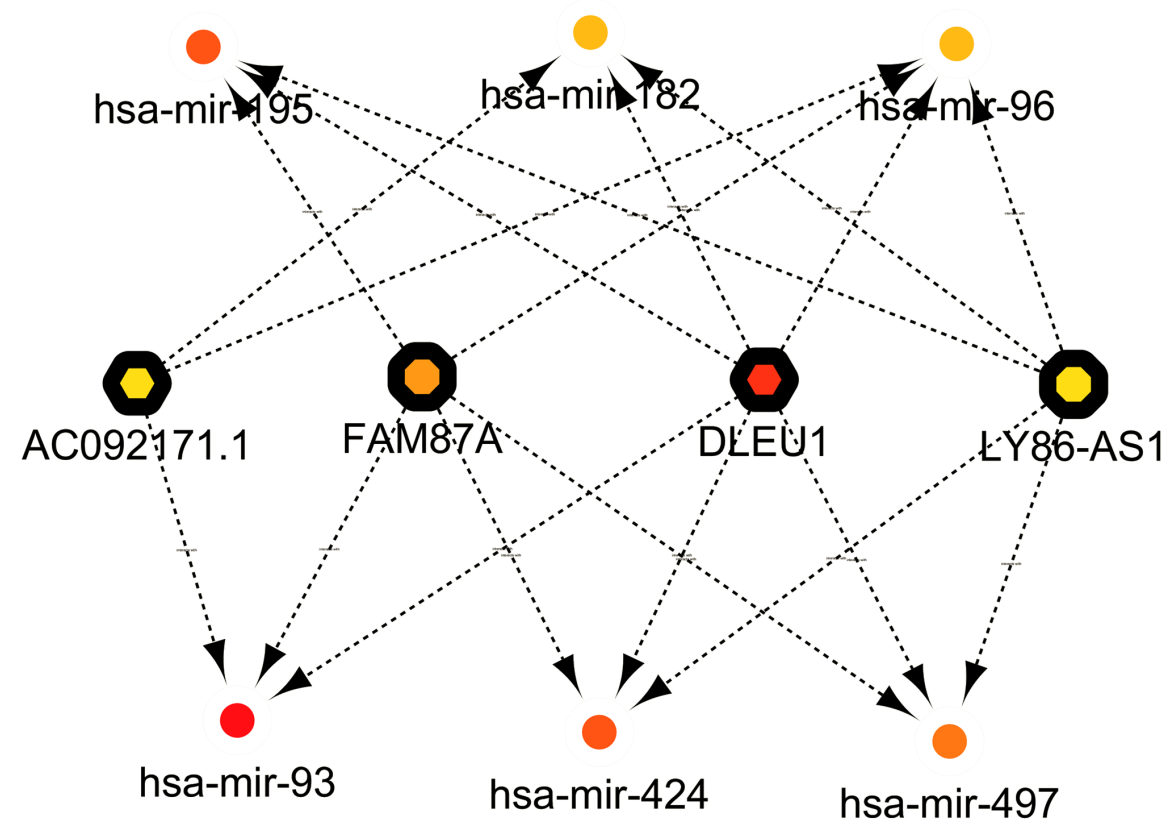

Figure 7. Network of top-10 based on closeness in the competing endogenous RNA network. The network consisted of 6 miRNAs, including hsa-mir-93, hsa-mir-424, hsa-mir-497, hsa-mir-195, hsa-mir-182 and hsa-mir-96 and 4 lncRNAs, FAM87A, family with sequence similarity 87 member A; DLEU1, deleted in lymphocytic leukemia 1; LY86-AS1, lymphocyte antigen 86 antisense RNA 1; AC092171.1, non-protein coding RNA. Boxes with rounded edges, miRNAs; boxes with hexagon edges, IncRNAs.

sarcoma-associated herpesvirus infection', 'relaxin signaling pathway' and 'human T-cell leukemia virus 1 infection'. Among the enriched pathways, 'Kaposi sarcoma-associated herpesvirus infection' has been reported to modulate the proliferation of glioma stem-like cells (39). Kaposi's sarcoma-associated herpesvirus exhibits neurotropism and its mRNA was detected in the plasma of patients with glioma in previous studies (39-41). Another previous study suggested that viral infection was a risk factor for glioma (42). The potential association between Kaposi's sarcoma-associated herpesvirus infection and glioma progression warrants further investigation. Among the DEmRNAs in the ceRNA network, tyrosine 3-monooxygenase/tryptophan 5-monooxygenase activation protein (YWHA)-H, E2F transcription factor (E2F) 5, E2F2, WEE1 $\mathrm{G}_{2}$ checkpoint kinase (WEE1), cell division cycle 25A (CDC25A) and checkpoint kinase 1 (CHEK1) were enriched in cell cycle-associated pathways. The present study demonstrated that YWHAH was significantly downregulated and associated with hsa-mir-424, hsa-mir-497 and hsa-mir-195 in the ceRNA network. A previous study demonstrated that YWHAH was involved in several cellular processes, including $\mathrm{G}_{1} / \mathrm{S}$ and $\mathrm{G}_{2} / \mathrm{M}$ cell cycle transition (43). Additionally, YWHAE, another YWHA isoform involved in cell cycle control and signal transduction $(44,45)$, was associated with copy number aberrations in astrocytoma pathogenesis (46). E2F5 is a key transcription factor involved in cell cycle progression. In a previous study, E2F5 silencing inhibited the proliferation of GBM cells and induced cell cycle arrest (47). miR-218 inhibited the growth and metabolism of glioma cells by targeting E2F2 (48). WEE1, a regulator of the $\mathrm{G}_{2}$ checkpoint in GBM cells, was associated with malignancy and poor outcomes of patients with GBM (49). CDC25A and CHEK1 are significant regulators of the cell cycle and apoptosis in glioma cells (50).
The top 10 RNAs in the ceRNA network were identified using the cytohubba plugin. The network consisted of 6 miRNAs, including hsa-mir-93, hsa-mir-424, hsa-mir-497, hsa-mir-195, hsa-mir-182 and hsa-mir-96, and 4 lncRNAs, including AC092171.1, FAM87A, DLEU1 and LY86-AS1. LY86-AS1 and FAM87A were demonstrated to have a high degree of closeness with hsa-mir-424, hsa-mir-497 and hsa-mir-195. A previous study revealed that hsa-mir-497 demonstrated high sensitivity and specificity for the prediction of malignant astrocytomas (51). Moreover, overexpression of hsa-mir-497 promoted the proliferation of U87 glioma cells by targeting neuregulin receptor degradation protein 1 (37). Previous studies held conflicting views on the role of aberrantly expressed hsa-mir-195 in gliomas. For instance, hsa-mir-195 was demonstrated to inhibit the proliferation of human glioma cells by directly targeting cyclin D1 and cyclin E1 mRNA (52), and was positively correlated with the OS of patients with GBM (53). Additionally, Zhang et al (54) revealed that overexpression of hsa-mir-195 resulted in cell cycle arrest and significantly decreased invasion of GBM cell lines. By contrast, hsa-mir-195 promoted the proliferation and invasion of the human glioma cell line U87 via the transforming growth factor- $\beta$ (TGF- $\beta$ ) signaling pathway (55). The present results are in agreement with those reported by Zhang et al (54) and suggested that hsa-mir-195 was upregulated in glioma tissues. The mechanisms underlying the interactions between hsa-mir-195, YWHAH and other potential mRNAs involved in cell cycle regulation require further investigation.

Protein-coding genes have long been recognized as conventional cancer biomarkers. Nevertheless, IncRNAs, which play a major role in the regulation of gene expression, have become the focus of predictive biomarker investigation due to their ability to better reflect tumor prognosis (11). Accumulating evidence suggests that dysregulation of IncRNA expression 
Table I. GO pathways enriched with differentially expressed mRNA involved in the competing endogenous RNA network.

GO ID Term Genes P-value

$0005515 \quad$ Protein binding

0051726 Regulation of cell cycle

$0004672 \quad$ Protein kinase activity

$0004713 \quad$ Protein tyrosine kinase activity

$0004871 \quad$ Signal transducer activity
E2F2, LIMA1, E2F5, CPEB3, TOLLIP, TLN2, PDLIM5, E2F7, $\quad<0.001$ TSPAN6, CBX2, ZEB1, CBFA2T3, MMP2, YBX1, DAB2, MAP3K9, RAPGEF4, RHOC, TMEM100, GNG5, CDCA4, NRIP3, PPP1R37, KIF5C, PRKCE, WEE1, BTN3A1, TRIM36, BTG1, TPPP, RRM2, GNB5, MAPK9, MAPRE3, PPP3R1, SOX4, BCL2L2, CHEK1, CEP55, ABCA1, ATP6V1B2, HPRT1, FBXW7, MOAP1, BCL11B, ETV1, SSX2IP, POLQ, TXNIP, GABARAPL1, ADARB1, MAP2K1, MAP2K4, ELAVL2, MAFK, CDC25A, ITPR1, EPHA4, EPHA7, YWHAH, DUSP2, SLC16A9, FAM126B, PLEKHA1, TP53INP1 E2F2, ADARB1, TRIM36, E2F5, CDC25A, WEE1 $<0.001$ EPHA4, MAP2K1, MAP3K9, MAP2K4, STK17B, MAPK9, $\quad<0.001$ CHEK1, PRKCE, WEE1

EPHA4, EPHA7, MAP2K1, MAP3K9, MAP2K4, WEE1 $<0.001$ GNAL, TOLLIP, TSPAN6, GNB5, RHOC, PRKCE, GNG5 $\quad<0.001$

GO, Gene Ontology.

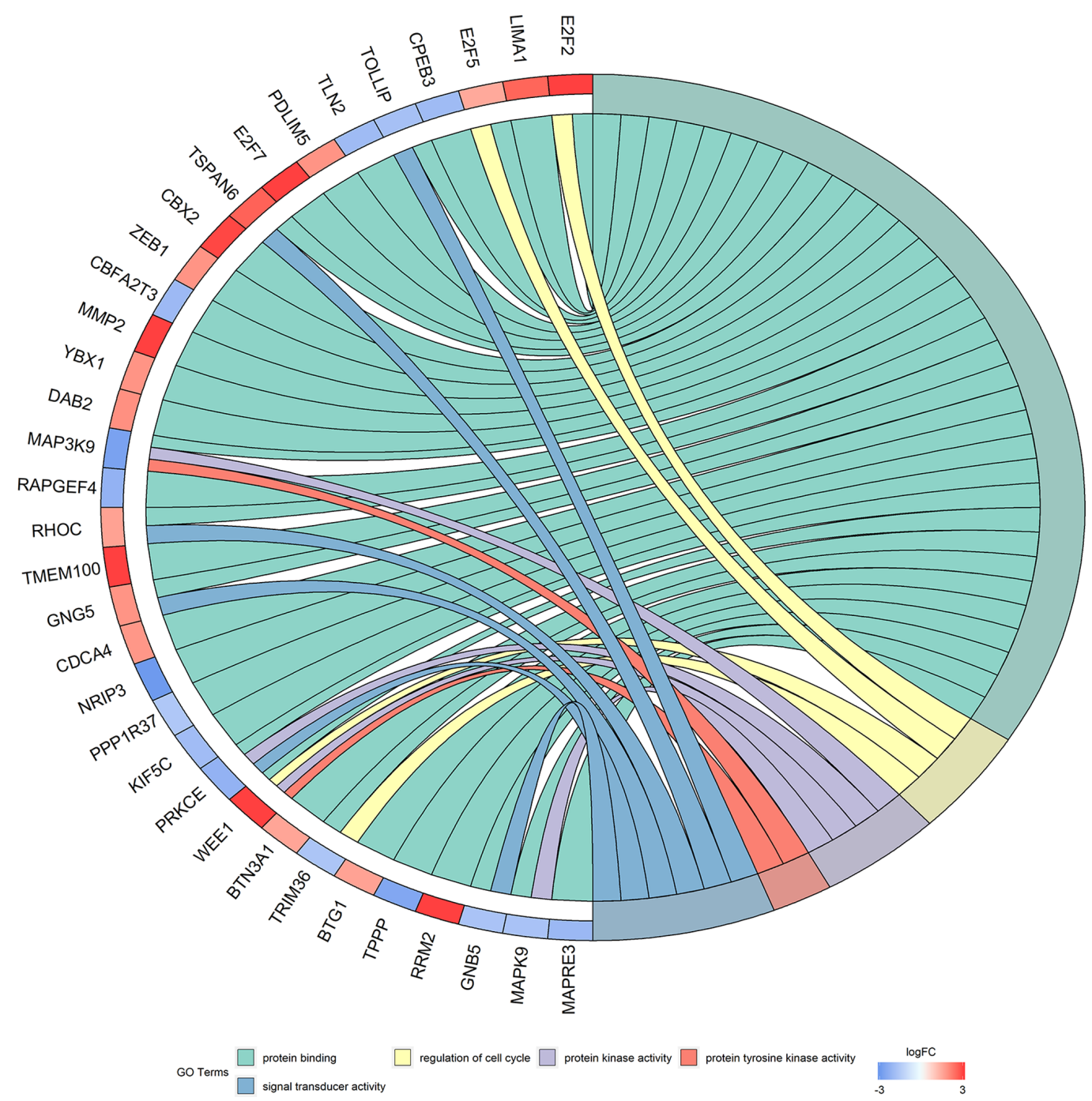

Figure 8. Enriched GO biological process terms of differentially expressed mRNAs involved in the competing endogenous RNA network. GO, Gene Ontology; FC, fold change. 
Table II. Kyoto Encyclopedia of Genes and Genomes pathways enriched with differentially expressed mRNA involved in the competing endogenous RNA network.

\begin{tabular}{|c|c|c|c|c|}
\hline Pathway ID & Description & Genes & Count & P-value \\
\hline hsa05167 & $\begin{array}{l}\text { Kaposi sarcoma-associated } \\
\text { herpesvirus infection }\end{array}$ & $\begin{array}{l}\text { MAP2K1, MAPK9, PPP3R1, MAP2K4, GABARAPL1, } \\
\text { GNB5, ITPR1, E2F2, GNG5 }\end{array}$ & 9 & $<0.001$ \\
\hline hsa05170 & $\begin{array}{l}\text { Human immunodeficiency } \\
\text { virus } 1 \text { infection }\end{array}$ & $\begin{array}{l}\text { MAP2K1, MAPK9, PPP3R1, GNB5, ITPR1, WEE1, } \\
\text { GNG5, CHEK1 }\end{array}$ & 8 & $<0.001$ \\
\hline hsa04218 & Cellular senescence & $\begin{array}{l}\text { MAP2K1, PPP3R1, ITPR1, E2F5, E2F2, CDC25A, } \\
\text { CHEK1 }\end{array}$ & 7 & $<0.001$ \\
\hline hsa04110 & Cell cycle & YWHAH, E2F5, E2F2, WEE1, CDC25A, CHEK1 & 6 & $<0.001$ \\
\hline hsa04926 & Relaxin signaling pathway & MAP2K1, MAPK9, MAP2K4, GNB5, MMP2, GNG5 & 6 & $<0.001$ \\
\hline hsa04728 & Dopaminergic synapse & MAPK9, GNB5, ITPR1, KIF5C, GNG5, GNAL & 6 & $<0.001$ \\
\hline hsa04371 & Apelin signaling pathway & MAP2K1, GABARAPL1/GNB5, PRKCE, ITPR1, GNG5 & 6 & $<0.001$ \\
\hline hsa04912 & GnRH signaling pathway & MAP2K1, MAPK9, MAP2K4, ITPR1, MMP2 & 5 & $<0.001$ \\
\hline hsa05166 & $\begin{array}{l}\text { Human T-cell leukemia } \\
\text { virus } 1 \text { infection }\end{array}$ & $\begin{array}{l}\text { MAP2K1, MAPK9, PPP3R1, MAP2K4, TLN2, E2F2, } \\
\text { CHEK1 }\end{array}$ & 7 & $<0.001$ \\
\hline
\end{tabular}

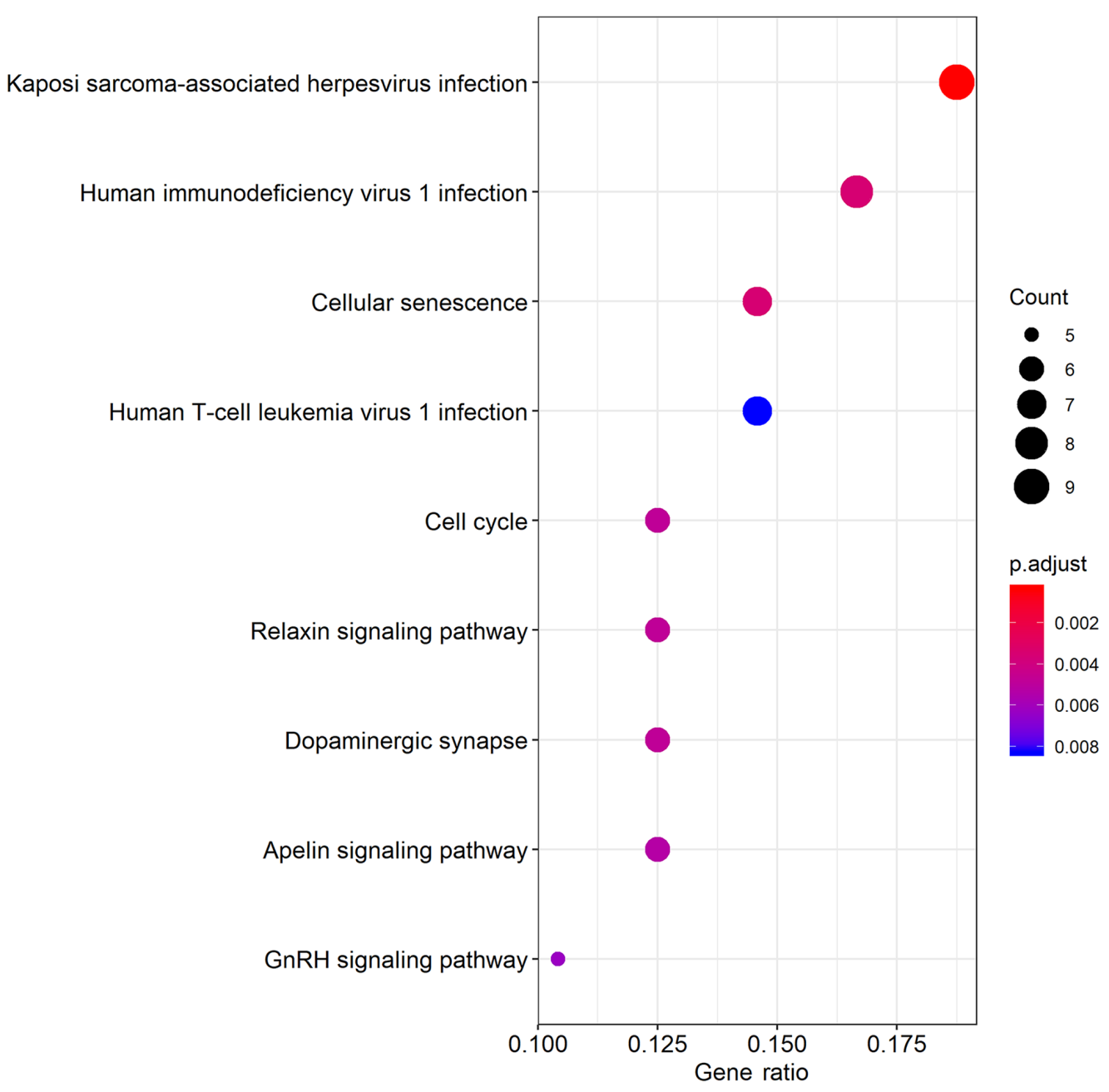

Figure 9. Top-9 enriched Kyoto Encyclopedia of Genes and Genomes pathway of differentially expressed mRNAs involved in the competing endogenous RNA network.

is associated with carcinogenesis and progression of glioma. Therefore, IncRNAs may serve as potential biomarkers for prognosis (56-58). For instance, colorectal neoplasia differentially expressed (CRNDE), the most upregulated lncRNA in glioma (59), facilitated cancer cell proliferation, migration and invasion by downregulating miR-384 expression. 



Figure 10. Kaplan-Meier survival curves of 6 differentially expressed long non-coding RNAs associated with overall survival in glioma. Overall survival curves of (A) DLEU1, (B) DBH-AS1, (C) HAS2-AS1, (D) LINC00320, (E) MEG8 and (F) MIR600HG. DLEU1, deleted in lymphocytic leukemia 1; DBH-AS1, dopamine $\beta$-hydroxylase antisense RNA1; HAS2-AS1, hyaluronan synthase 2 antisense RNA 1; LINC00320, long intergenic non-protein coding RNA 320; MEG8, maternally expressed 8.

CRNDE knockdown combined with miR-384 overexpression significantly attenuated tumor progression in vivo (57). IncRNA activated by TGF- $\beta$ (IncRNA-ATB) served as a ceRNA and a sponge for miR-200a, promoting cell proliferation and invasion in glioma. Upregulation of IncRNA-ATB was associated with worse OS in patients with glioma (60).

In the present study, 92 DElncRNAs were involved in the ceRNA network. Kaplan-Meier curve analysis indicated that 36 DElncRNAs were associated with OS. Therefore, these lncRNAs may serve as potential prognostic biomarkers in patients with glioma. Tian et al demonstrated that LINC00320, a tumor-suppressive IncRNA, was downregulated in glioma tissues and inhibited the proliferation of glioma cells by repressing the $\mathrm{Wnt} / \beta$-catenin signaling pathway in vitro and in vivo (26). Moreover, Mills et al (61) demonstrated that full-length LINC00320 was only expressed in human brain tissue, suggesting that it may be a highly specific biomarker for glioma. Zhu et al (62) have demonstrated that the expression level of HAS2-AS1 was closely associated with lymph node metastasis and hypoxic tumor status in patients with oral squamous cell carcinoma. Notably, the expression level of HAS2-AS1 was negatively associated with OS in the present study. DLEU1 was identified to act as a sponge for miR-490 and contributed to the development of endometrial cancer (63). Furthermore, DLEU1 has been widely accepted as an oncogenic lncRNA and its aberrant upregulation was associated with the outcome of various types of cancer, including gastric (64), pancreatic (65) and colorectal cancer (66). Terashima et al (67) demonstrated that MEG8 was required for the epithelial-mesenchymal transition in lung and pancreatic cancer cells. DBH-AS1 was demonstrated to promote cell proliferation and survival through the MAPK signaling pathway in hepatocellular carcinoma cells (68). MIR600HG independently correlated with the outcome of patients with pancreatic cancer (69). In the present study, low MEG8 and DBH-AS1 expression, as well as high MIR600HG expression, were associated with an improved outcome. Therefore, further investigation is required to validate whether these IncRNAs may serve as potential predictive biomarkers for glioma.

However, there were some limitations in the present study. Indeed, the present study was designed as a bioinformatics analysis based on a small sample size, which also lacked experimental verification. Further experiment validation in larger samples is required to verify the present findings.

In conclusion, the present study constructed a specific ceRNA network consisting of lncRNAs, miRNAs and mRNAs, and the mechanisms of ceRNA regulation were further analyzed. Survival analysis indicated that lncRNAs may serve as potential biomarkers for predicting the outcomes of patients with glioma. To the best of the authors' knowledge, the present study was the first to attempt to construct a specific ceRNA network and to investigate the interactions between miRNAs and lncRNAs in glioma based on TCGA data. 


\section{Acknowledgements}

Not applicable.

\section{Funding}

The present study was supported by The Science and Education for Health Foundation of Suzhou for Youth (grant nos. kjxw2018030 and kjxw2018032), The Science and Technology Project Foundation of Suzhou (grant no. SS201651), The Medical Key Discipline Foundation of Jiangsu Province (grant no. ZDXKC2016007) and The Education Research Project Foundation of Nanjing Medical University (grant no. FZS-ZD-201701).

\section{Availability of data and materials}

The datasets used and/or analyzed during the present study are available from the corresponding author on reasonable request.

\section{Authors' contributions}

ZLD and WJW contributed to the conception and design of the study. JZ and YQH performed data analysis and curation. HW performed the statistical analysis. WS and YFL carried out the concept, design, definition of intellectual content, manuscript preparation and wrote the first draft of the manuscript. All authors read and approved the final manuscript.

\section{Ethics approval and consent to participate}

Not applicable.

\section{Patient consent for publication}

Not applicable.

\section{Competing interests}

The authors declare that they have no competing interests.

\section{References}

1. Ostrom QT, Gittleman H, Truitt G, Boscia A, Kruchko C and Barnholtz-Sloan JS: CBTRUS statistical report: Primary brain and other central nervous system tumors diagnosed in the United States in 2011-2015. Neuro Oncol 20 (Suppl 4): iv1-iv86, 2018.

2. Vigneswaran K, Neill S and Hadjipanayis CG: Beyond the World Health Organization grading of infiltrating gliomas: Advances in the molecular genetics of glioma classification. Ann Trans Med 3: 95, 2015.

3. National Brain Tumor Association. Quick brain tumor facts 2018. Available at: http: //braintumor.org/brain-tumor-information/brain-tumor-facts/(accessed November 27, 2017)

4. American Brain Tumor Association (ABTA). Brain tumor statistics; 2017. Available at: http://www.abta.org/about-us/ news/brain-tumor-statistics/. Accessed 22 November, 2017.

5. McNeill KA: Epidemiology of brain tumors. Neurol Clin 34: 981-998, 2016

6. Barone F, Alberio N, Iacopino DG, Giammalva GR, D'Arrigo C Tagnese W, Graziano F, Cicero S and Maugeri R: Brain mapping as helpful tool in brain glioma surgical treatment-toward the ‘Perfect Surgery'? Brain Sci 8: 192, 2018.
7. Ostrom QT, Gittleman H, Liao P, Vecchione-Koval T, Wolinsky Y, Kruchko C and Barnholtz-Sloan JS: CBTRUS statistical report: Primary brain and other central nervous system tumors diagnosed in the United States in 2010-2014. Neuro Oncol 19 (Suppl 5): v1-v88, 2017.

8. Venur VA, Peereboom DM and Ahluwalia MS: Current medical treatment of glioblastoma. Cancer Treat Res 163: 103-115, 2015.

9. Kang JH and Adamson C: Novel chemotherapeutics and other therapies for treating high-grade glioma. Expert Opin Investig Drugs 24: 1361-1379, 2015.

10. Salmena L, Poliseno L, Tay Y, Kats L and Pandolfi PP: A ceRNA hypothesis: The Rosetta Stone of a hidden RNA language? Cell 146: 353-358, 2011

11. Hauptman $\mathrm{N}$ and Glavač D: Long non-coding RNA in cancer. Int J Mol Sci 14: 4655-4669, 2013.

12. Mercer TR, Dinger ME and Mattick JS: Long non-coding RNAs: Insights into functions. Nat Rev Genet 10: 155-159, 2009.

13. Sotillo $\mathrm{E}$ and Thomas-Tikhonenko A: The long reach of noncoding RNAs. Nat Genet 43: 616-617, 2011.

14. Mercer TR and Mattick JS: Structure and function of long noncoding RNAs in epigenetic regulation. Nat Struct Mol Biol 20: 300-307, 2013.

15. Tay Y, Rinn J and Pandolfi PP: The multilayered complexity of ceRNA crosstalk and competition. Nature 505: 344-352, 2014.

16. Peng Y and Croce CM: The role of MicroRNAs in human cancer. Signal Transduct Target Ther 1: 15004, 2016.

17. Liu J, Lichtenberg T, Hoadley KA, Poisson LM, Lazar AJ, Cherniack AD, Kovatich AJ, Benz CC, Levine DA, Lee AV, et al: An integrated TCGA pan-cancer clinical data resource to drive high-quality survival outcome analytics. Cell 173: 400-416.e11,2018.

18. Anders S and Huber W: Differential expression analysis for sequence count data. Genome Biol 11: R106, 2010.

19. Fromm B, Billipp T, Peck LE, Johansen M, Tarver JE, King BL, Newcomb JM, Sempere LF, Flatmark K, Hovig E and Peterson KJ: A uniform system for the annotation of vertebrate microRNA genes and the evolution of the human microRNAome. Annu Rev Genet 49: 213-242, 2015.

20. Chou CH, Chang NW, Shrestha S, Hsu SD, Lin YL, Lee WH, Yang CD, Hong HC, Wei TY, Tu SJ, et al: miRTarBase 2016: Updates to the experimentally validated miRNA-target interactions database. Nucleic Acids Res 44 (D1): D239-D247, 2016.

21. Wong N and Wang X: miRDB: An online resource for microRNA target prediction and functional annotations. Nucleic Acids Res 43 (Database issue): D146-D152, 2015.

22. Chin CH, Chen SH, Wu HH, Ho CW, Ko MT and Lin CY: cytoHubba: Identifying hub objects and sub-networks from complex interactome. BMC Syst Biol 8 (Suppl 4): S11, 2014

23. Dennis G Jr, Sherman BT, Hosack DA, Yang J, Gao W, Lane HC and Lempicki RA: DAVID: Database for annotation, visualization, and integrated discovery. Genome Biol 4: P3, 2003.

24. Chan JJ and Tay Y: Noncoding RNA:RNA regulatory networks in cancer. Int J Mol Sci 19: 1310, 2018.

25. Wang X, Arai S, Song X, Reichart D, Du K, Pascual G, Tempst P, Rosenfeld MG, Glass CK and Kurokawa R: Induced ncRNAs allosterically modify RNA-binding proteins in cis to inhibit transcription. Nature 454: 126-130, 2008.

26. Tian S, Liu W, Pan Y and Zhan S: Long non-coding RNA Linc00320 inhibits glioma cell proliferation through restraining Wnt/ $\beta$-catenin signaling. Biochem Biophys Res Commun 508: 458-464, 2019.

27. Liu C, Liu R, Zhang D, Deng Q, Liu B, Chao HP, Rycaj K, Takata Y, Lin K, Lu Y, et al: MicroRNA-141 suppresses prostate cancer stem cells and metastasis by targeting a cohort of pro-metastasis genes. Nat Commun 8: 14270, 2017.

28. Calin GA and Croce CM: MicroRNA signatures in human cancers. Nat Rev Cancer 6: 857-866, 2006.

29. Shi L, Zhang J, Pan T, Zhou J, Gong W, Liu N, Fu Z and You Y: MiR-125b is critical for the suppression of human U251 glioma stem cell proliferation. Brain Res 1312: 120-126, 2010.

30. Wang L, Shi ZM, Jiang CF, Liu X, Chen QD, Qian X, Li DM, Ge X, Wang XF, Liu LZ, et al: MiR-143 acts as a tumor suppressor by targeting N-RAS and enhances temozolomide-induced apoptosis in glioma. Oncotarget 5: 5416-5427, 2014.

31. Xu X, Bao Z, Liu Y, Ji J and Liu N: MicroRNA-98 attenuates cell migration and invasion in glioma by directly targeting Pre-B cell leukemia homeobox 3. Cell Mol Neurobiol 37: 1359-1371, 2017.

32. Anwar SL,Krech T,Hasemeier B, Schipper E, Schweitzer N, Vogel A, Kreipe H, Buurman R, Skawran B and Lehmann U: hsa-mir-183 is frequently methylated and related to poor survival in human hepatocellular carcinoma. World J Gastroenterol 23: 1568-1575, 2017. 
33. Zhang BL, Dong FL, Guo TW, Gu XH, Huang LY and Gao DS: MiRNAs mediate GDNF-induced proliferation and migration of glioma cells. Cell Physiol Biochem 44: 1923-1938, 2017.

34. Li HY, Li YM, Li Y, Shi XW and Chen H: Circulating microRNA-137 is a potential biomarker for human glioblastoma. Eur Rev Med Pharmacol Sci 20: 3599-3604, 2016.

35. Yang W, Bai J, Liu D, Wang S, Zhao N, Che R and Zhang H: MiR-93-5p up-regulation is involved in non-small cell lung cancer cells proliferation and migration and poor prognosis Gene 647: 13-20, 2018.

36. Li HY, Liang JL, Kuo YL, Lee HH, Calkins MJ, Chang HT, Lin FC, Chen YC, Hsu TI, Hsiao M, et al: miR-105/93-3p promotes chemoresistance and circulating miR-105/93-3p acts as a diagnostic biomarker for triple negative breast cancer. Breast Cancer Res 19: 133, 2017.

37. Gao Y, Sun L, Wu Z and Chen X: Over-expression of miR-497 promotes the proliferation of U87 glioma cells by targeting neuregulin receptor degradation protein 1 . Xi Bao Yu Fen $\mathrm{Zi}$ Mian Yi Xue Za Zhi 33: 1051-1055, 2017 (In Chinese).

38. Feng F, Kuai D, Wang H, Li T, Miao W, Liu Y and Fan Y: Reduced expression of microRNA-497 is associated with greater angiogenesis and poor prognosis in human gliomas. Hum Pathol 58: 47-53, 2016.

39. Jeon H, Kang YH, Yoo SM, Park MJ, Park JB, Lee SH and Lee MS: Kaposi's sarcoma-associated herpesvirus infection modulates the proliferation of glioma stem-like cells. J Microbiol Biotechnol 28: 165-174, 2018

40. Chan PK, Ng HK, Cheung JL and Cheng AF: Survey for the presence and distribution of human herpesvirus 8 in healthy brain. J Clin Microbiol 38: 2772-2773, 2000.

41. Merelli E, Bedin R, Sola P, Barozzi P, Mancardi GL, Ficarra G and Franchini G: Human herpes virus 6 and human herpes virus 8 DNA sequences in brains of multiple sclerosis patients, normal adults and children. J Neurol 244: 450-454, 1997.

42. McFaline-Figueroa JR and Wen PY: The viral connection to glioblastoma. Curr Infect Dis Rep 19: 5, 2017.

43. Lee CG, Park GY, Han YK, Lee JH, Chun SH, Park HY, Lim KH, Kim EG, Choi YJ, Yang K and Lee CW: Roles of $14-3-3 \eta$ in mitotic progression and its potential use as a therapeutic target for cancers. Oncogene 32: 1560-1569, 2013

44. Jin J, Smith FD, Stark C, Wells CD, Fawcett JP, Kulkarni S, Metalnikov P, O'Donnell P, Taylor P, Taylor L, et al: Proteomic, functional, and domain-based analysis of in vivo 14-3-3 binding proteins involved in cytoskeletal regulation and cellular organization. Curr Biol 14: 1436-1450, 2004

45. Mackintosh C: Dynamic interactions between 14-3-3 proteins and phosphoproteins regulate diverse cellular processes Biochem J 381: 329-342, 2004

46. Pećina-Šlaus N, Kafka A, Gotovac Jerčić K, Logara M, Bukovac A, Bakarić R and Borovečki F: Comparable genomic copy number aberrations differ across astrocytoma malignancy grades. Int J Mol Sci 20: 1251, 2019.

47. Xu X, Cai N, Zhi T, Bao Z, Wang D, Liu Y, Jiang K, Fan L, Ji J and Liu N: MicroRNA-1179 inhibits glioblastoma cell proliferation and cell cycle progression via directly targeting E2F transcription factor 5. Am J Cancer Res 7: 1680-1692, 2017.

48. Zhang Y, Han D, Wei W, Cao W, Zhang R, Dong Q, Zhang J, Wang Y and Liu N: MiR-218 inhibited growth and metabolism of human glioblastoma cells by directly targeting E2F2. Cell Mol Neurobiol 35: 1165-1173, 2015 .

49. Music D, Dahlrot RH, Hermansen SK, Hjelmborg J, de Stricker K, Hansen S and Kristensen BW: Expression and prognostic value of the WEE1 kinase in gliomas. J Neurooncol 127: 381-389, 2016.

50. Krell A, Wolter M, Stojcheva N, Hertler C, Liesenberg F, Zapatka M, Weller M, Malzkorn B and Reifenberger G: MiR-16-5p is frequently down-regulated in astrocytic gliomas and modulates glioma cell proliferation, apoptosis, and response to cytotoxic therapy. Neuropathol Appl Neurobiol 45: 441-458, 2019.

51. Yang C, Wang C, Chen X, Chen S, Zhang Y, Zhi F, Wang J, Li L, Zhou X, Li N, et al: Identification of seven serum microRNAs from a genome-wide serum microRNA expression profile as potential noninvasive biomarkers for malignant astrocytomas. Int J Cancer 132: 116-127, 2013.

52. Hui W, Yuntao L, Lun L, WenSheng L, ChaoFeng L, HaiYong H and Yueyang B: MicroRNA-195 inhibits the proliferation of human glioma cells by directly targeting cyclin D1 and cyclin E1. PLoS One 8: e54932, 2013.
53. Lakomy R, Sana J, Hankeova S, Fadrus P, Kren L, Lzicarova E, Svoboda M, Dolezelova H, Smrcka M, Vyzula R, et al: MiR-195, miR-196b, miR-181c, miR-21 expression levels and O-6-methylguanine-DNA methyltransferase methylation status are associated with clinical outcome in glioblastoma patients. Cancer Sci 102: 2186-2190, 2011.

54. Zhang QQ, Xu H, Huang MB, Ma LM, Huang QJ, Yao Q, Zhou H and Qu LH: MicroRNA-195 plays a tumor-suppressor role in human glioblastoma cells by targeting signaling pathways involved in cellular proliferation and invasion. Neuro Oncol 14: 278-287, 2012.

55. Duan Y and Chen Q: TGF- $\beta 1$ regulating miR-205/miR-195 expression affects the TGF- $\beta$ signal pathway by respectively targeting SMAD2/SMAD7. Oncol Rep 36: 1837-1844, 2016.

56. Wang Q, Zhang J, Liu Y, Zhang W, Zhou J, Duan R, Pu P, Kang C and Han L: A novel cell cycle-associated lncRNA, HOXA11-AS, is transcribed from the 5-prime end of the HOXA transcript and is a biomarker of progression in glioma. Cancer Lett 373: 251-259, 2016.

57. Zheng J, Liu X, Wang P, Xue Y, Ma J, Qu C and Liu Y: CRNDE promotes malignant progression of glioma by attenuating miR-384/PIWIL4/STAT3. Mol Ther 24: 1199-1215, 2016.

58. Brodie S, Lee HK, Jiang W, Cazacu S, Xiang C, Poisson LM, Datta I, Kalkanis S, Ginsberg D and Brodie C: The novel long non-coding RNA TALNEC2, regulates tumor cell growth and the stemness and radiation response of glioma stem cells. Oncotarget 8: 31785-31801, 2017.

59. Li DX, Fei XR, Dong YF, Cheng CD, Yang Y, Deng XF, Huang HL, Niu WX, Zhou CX, Xia CY and Niu CS: The long non-coding RNA CRNDE acts as a ceRNA and promotes glioma malignancy by preventing miR-136-5p-mediated downregulation of Bcl-2 and Wnt2. Oncotarget 8: 88163-88178, 2017.

60. Ma CC, Xiong Z, Zhu GN, Wang C, Zong G, Wang HL, Bian EB and Zhao B: Long non-coding RNA ATB promotes glioma malignancy by negatively regulating miR-200a. J Exp Clin Cancer Res 35: 90, 2016.

61. Mills JD, Chen J, Kim WS, Waters PD, Prabowo AS, Aronica E, Halliday GM and Janitz M: Long intervening non-coding RNA 00320 is human brain-specific and highly expressed in the cortical white matter. Neurogenetics 16: 201-213, 2015.

62. Zhu G, Wang S, Chen J, Wang Z, Liang X, Wang X, Jiang J, Lang $\mathrm{J}$ and $\mathrm{Li}$ L: Long noncoding RNA HAS2-AS1 mediates hypoxia-induced invasiveness of oral squamous cell carcinoma. Mol Carcinog 56: 2210-2222, 2017.

63. Shao W, Li Y, Chen F, Jia H, Jia J and Fu Y: Long non-coding RNA DLEU1 contributes to the development of endometrial cancer by sponging miR-490 to regulate SP1 expression. Pharmazie 73: 379-385, 2018.

64. Li X, Li Z, Liu Z, Xiao J, Yu S and Song Y: Long non-coding RNA DLEU1 predicts poor prognosis of gastric cancer and contributes to cell proliferation by epigenetically suppressing KLF2. Cancer Gene Ther 25: 58-67, 2018.

65. Gao S, Cai Y, Zhang H, Hu F, Hou L and Xu Q: Long noncoding RNA DLEU1 aggravates pancreatic ductal adenocarcinoma carcinogenesis via the miR-381/CXCR4 axis. J Cell Physiol 234: 6746-6757, 2019

66. Liu T, Han Z, Li H, Zhu Y, Sun Z and Zhu A: LncRNA DLEU1 contributes to colorectal cancer progression via activation of KPNA3. Mol Cancer 17: 118, 2018.

67. Terashima M, Ishimura A, Wanna-Udom S and Suzuki T: MEG8 long noncoding RNA contributes to epigenetic progression of the epithelial-mesenchymal transition of lung and pancreatic cancer cells. J Biol Chem 293: 18016-18030, 2018.

68. Huang JL, Ren TY, Cao SW, Zheng SH, Hu XM, Hu YW, Lin L, Chen J, Zheng L and Wang Q: HBx-related long non-coding RNA DBH-AS1 promotes cell proliferation and survival by activating MAPK signaling in hepatocellular carcinoma. Oncotarget 6: 33791-33804, 2015 .

69. Song J, Xu Q, Zhang H, Yin X, Zhu C, Zhao K and Zhu J: Five key lncRNAs considered as prognostic targets for predicting pancreatic ductal adenocarcinoma. J Cell Biochem 119 4559-4569, 2018.

This work is licensed under a Creative Commons Attribution-NonCommercial-NoDerivatives 4.0 International (CC BY-NC-ND 4.0) License. 